\title{
The Personal Health Record
}

\section{Jeongeun Kim, $\mathrm{PhD}$}

College of Nursing, Seoul National University, Seoul, Korea

\section{The Personal Health Record}

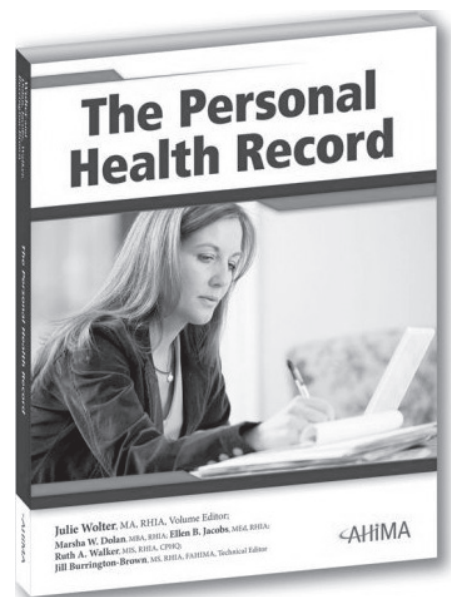

Authors: Julie Wolter, Marsha W. Dolan, Ellen B. Jacobs, Rush A. Walker, and Jill Burrington-Brown Year: 2009

Publisher: AHIMA (American Health Information Management Association) Press ISBN-10: 1584262060 ISBN-13: 978-1584262060 Product Dimensions: $10 \times 7.9 \times 0.3$ inches Paperback 152 pages Price: $\$ 54.95$

\section{Personal Health Records: The Essential Missing Element in 21st Century Health- care}



Authors: Holly Dara Miller, William A. Yasnoff, and Howard A. Burde

Year: 2009

Publisher: HIMSS (Health Information Management Systems Society) ISBN: 978-0-9800697-6-1 Softcover 178 pages

Price: $\$ 72$

This is an Open Access article distributed under the terms of the Creative Commons Attribution Non-Commercial License (http://creativecommons.org/licenses/bync/3.0/) which permits unrestricted non-commercial use, distribution, and reproduction in any medium, provided the original work is properly cited.

(C) 2011 The Korean Society of Medical Informatics

\section{Personal Health Record: A Guide for Clinicians}

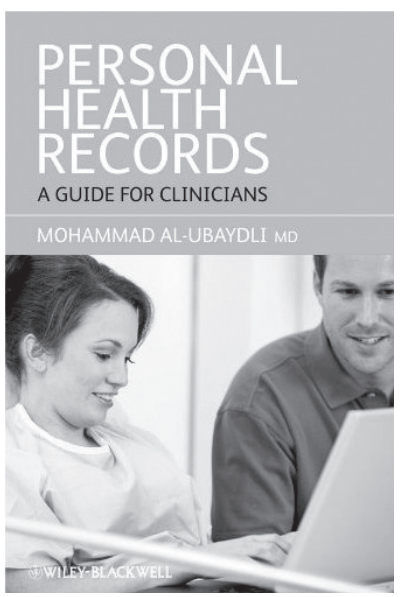

Author: Mohammad Al-Ubaydli, MD

Year: 2011

Publisher: Wiley-Blackwell, ISBN-10: 144433252X

ISBN-13: 978-1444332520

Product Dimensions:

$9.1 \times 6.1 \times 0.4$ inches

Paperback 136 pages

Price: $\$ 39.95$

Many healthcare information technology (HIT) groups have demonstrated a growing interest in PHRs. Citing some examples of this interest among many are HIMSS' inauguration of a PHR Steering Committee in 2006; HL7 developing PHR related use cases in 2007; The Markle Foundation convening a group of industry experts regarding PHRs in 2007; CCHIT assuming the task of certifying PHRs, with a major focus on PHR privacy and security; and AHIC devoting the majority of a meeting to mobilizing consumers' personal health information, from both the consumer and industry perspectives. Several HIT groups have published PHR definitions, including the Markle Foundation, HIMSS and AMIA, and most recently NAHIT [1].

For the purpose of the Book Review on this topic, the author would present two books under the same name on similar period by representative associations (AHIMA \& HIMSS) in Medical Informatics discipline, and one book from the perspectives of a clinician which means that Personal Health Record is one of the main issues inside the scientific community and along the roadmap towards the perfect lifetime Electronic Health Record. 


\section{The Personal Health Record}

This book is designed to help HIM practitioners educate and guide consumers in creating PHRs. It provides an overview of why the PHR is needed and its relevance to the HIM professional. And it presents values and concerns of HIM professionals, provider organizations, physicians, consumers, and other parties. The book, also provides PHR models and HIM education for consumers, explains the benefits of PHRs to consumers, and explores the PHR's future [2].

The book consists of 3 main parts.

The first part, Industry Overview, includes four chapters: Chapter 1, Evolution of the Personal Health Record, introduces an overview and definition of PHR formulated by the organizations such as Connecting for Health and AHIMA. This chapter also provides the information about the type of PHRs, and what a PHR should contain. Chapter 2, Benefits and Concerns among Stakeholders, is about the benefits, opportunities, and concerns held by stakeholders in healthcare and how they view the PHR. This book addresses 9 stakeholders. Among them, those cases of most interests are of HIM professionals, provider organizations (and facilities), physicians (and physician groups), health insurance companies, employers. Chapter 3, New Roles and Potential Effects for the Health Information Management Professional, shows the role and effects for the HIM professionals. Especially, this chapter includes a list of some basic questions that consumers should consider in choosing a PHR, $<12$ questions consumers should ask when choosing a $P H R>$, which is developed by AHIMA Personal Health Record Practice Council. Chapter 4, Personal Health Record Models, describes of several major models for PHRs. There are some kind of Stand-Alone model, such as paper, personal computers, mobile storage devices, and web/internet storage. Other sorts of models include Integrated Models, which can be either tethered or untethered. Meanwhile, the term "tethered" means that the PHR connects with a single provider-based electronic health record (EHR) system or other institutional database.

The second part, The Consumer Perspective, consists of two chapters. Chapter 5, The Consumer and the Personal Health Record, shows the attributes of consumers "behaviors" in healthcare by Dr. Hibbard. Chapter 6, HIM Education for the Consumer, provides the information for HIM professionals about developing and using PHRs. The main context is about how to complete the PHR, quality of data, and sources of information. Also, it suggests that the PHR, being a consumer repository of health information, should contain the information about quality of care, home monitoring, diet, exercise, immunization, family history, telemedicine and re- mote monitoring of chronic conditions.

The third part, Personal Health Records and the Future, has only one chapter. Chapter 7, The Future of Personal Health Records, explains the paradigm shift for providers and consumers. Also, it contains lists of attributes that the PHR should possess in order to be "The Perfect PHR."

According to Jeremy Nobel, MD, MPH, the perfect PHR would [3]:

- Have an easily accessible and navigable Web site that displays a range of personalized health information

- Deliver personal clinical information accompanied by and linked to useful and culturally sensitive educational programs

- Offer links to healthcare providers who can help the consumer enhance his or her health

- Serve as a communications hub for consumers, physicians, nurses, health coaches, and advocates to securely exchange messages

- Compile health data and aggregate it in a way that fosters an understanding of best practices.

David Lansky, MD also has a list of attributes the PHR should have [4]:

- The ability to easily access emergency information

- The ability to track information and provide decision support

- The ability to shorten or lessen the time it takes to fill out forms and find important information

- The ability to connect to the Internet to research providers and literature

- The ability to track healthcare costs

- The ability to easily send and receive healthcare information to and from providers

- The ability to protect the privacy of the PHR from unauthorized users

Above this, this book points out the contexts about position papers, consumer management of financial health as an analogy for consumer management of Personal Health, caregivers managing patient records, records necessary for travel, and sample Personal Health Record and related forms as appendices.

\section{Personal Health Records: The Essential Missing Element in $21^{\text {st }}$ Century Healthcare}

In 2007, HIMSS requested the professionals of PHR to write a book on that issue. This book is meant to serve as a primer and a vision for PHRs. It is intended for readers who desire a basic introduction to the concepts, principles, and short history of this rapidly evolving area of health IT. 
"Personal Health Records" provides a comprehensive overview and discussion of the many issues pertaining to the adoption and use of personal health records, a rapidly evolving and essential area of healthcare IT. The book covers issues such as privacy, confidentiality and security; predictive modeling, Web 2.0 and social networking; the new patientprovider paradigm; public trust; applicable laws; and PHR business and sustainability models. The book presents practical implementation guidelines, plus project planning and budgeting information, and provides perspectives of various stakeholders including consumers, providers and payors [5]. This book consists of 10 chapters as follows:

Chapter 1. Need for ePHRs to Address Critical Problems in U.S. Healthcare

Chapter 2. Personal Health Record: History and Context

Chapter 3. Definitions, Models and Functions of ePHRs, PBHRs, EMRs and EHRs

Chapter 4. Evolving Market Forces Driving the Need for ePHRs

Chapter 5. Physicians, Patients and PHRs

Chapter 6. PHR Architectures

Chapter 7. Planning and Implementation of Healthcare Con-

stituent-Based PHRs: Some Practical Considerations

Chapter 8. PHR Law

Chapter 9. PHR Business Sustainability Models

Chapter 10. Conclusions

This book was fully translated into Korean and published in 2010 for professionals seriously interested in the PHR research and development by Jeongeun Kim, PhD \& Sukwha Kim, MD, PhD.

\section{Personal Health Record: A Guide for Cli- nicians}

Patient-controlled personal health records are the key to successful interaction between physician and patient. They form the core for joined-up communication throughout health organizations. Still, the very name is capable of alarming both patient and doctor. Are they reliable? Are they complete? Are they confidential? Where do you access them?

For the doctor, additional concerns surround the implementation: how do physicians include these online tools in their busy schedule? How much will PHRs add to physicians' existing spend on information technology? And can they get paid for doing all this extra work? This book puts the focus on these questions and tries to provide dependable answers to all of these questions. Written by a physician who has developed his own personal health records software for patients and doctors to interact, "Personal Health Records: A
Guide for Clinicians" explains how to get the best from patient's records and how to put the information to good use, helping both patients and physicians to a more effective and efficient outcome in any clinical situation [6]. The author is a clinical academic, patient and pioneer in his field and does a grand job of explaining the ins and outs of PHRs in a nonpatronising manner for the non-tech savvy [7].

This book consists of 5 chapters as follows;

Chapter 1. What is a PHR?

- What is a PHR? Why should you use one? How should you use it? How much does it cost? How can you get paid to use it?

Chapter 2. Your patients

- Sharing data with your patient: How do you share data with a patient? When should you not share data? What data should patients share with you?

- Protecting your patient's privacy: Once you have given out data about your patient, you can never take it back. How to be sure that the person you are giving data is the person who they say they are. And how to confirm that the patient is happy for you to give data to this person.

- Patient communities: How to participate in online communities for patients. How to promote your services to these patients. How to teach these patients. How to learn from these patients. How to conduct academic research online.

Chapter 3. Your work

- PHRs and clinical teams: PHRs work best when the whole clinical team uses them, and when the patient is part of that team. How to train your staff. How to allocate responsibilities to team members. How to maximize efficiency and minimize duplication. How to maintain and improve safety.

- Educating patients: Patients must learn a lot before they can understand their PHR. How to minimize the time you need to spend teaching. How to identify resources to refer your patient to. How to protect patients from incorrect information.

- Saving time in your clinic: How to save time by reducing face-to-face visits and phone calls.

Chapter 4. Your practice

- Technology: What hardware, software and data to buy. How to connect your patient's PHR to your clinic's EHR.

- Law: HIPAA, Data Protection Act, and other laws that apply to PHRs. How to protect yourself from litigation.

- Finance: How to get paid for using PHRs.

Chapter 5. The future

- Discuss other users of PHRs, e.g. researchers. 


\section{References}

1. Miller HD, Yasnoff WA, Burde HA. Personal health records: the essential missing element in 21st century healthcare. Chicago, IL: Healthcare Information and Management Systems Society; 2009.

2. American Health Information Management Association. Book: the personal health records [Internet]. Chicago, IL: American Health Information Management Association; c2011 [cited at 2011 Jun 1]. Available from: https://www.ahimastore.org/ProductDetailBooks. aspx?ProductID=12497.

3. Nobel JJ. The public health perspective. In: Gearon CJ, ed. Perspectives on the future of personal health records [Internet]. Oakland, CA: California Health Care Foundation; 2007 [cited at 2011 Jun 1]. p22-24. Available from: http://www.chcf.org/documents/chronicdisease/ PHRPerspectives.pdf.

4. Lansky D. The consumer perspective. In: Gearon CJ, ed. Perspectives on the future of personal health records [Internet]. Oakland, CA: California Health Care Foundation; 2007 [cited at 2011 Jun 1]. p10-13. Available from: http://www.chcf.org/documents/chronicdisease/ PHRPerspectives.pdf.

5. Healthcare Information and Management Systems Society. HIMSS store. Personal health records: the essential missing element in 21 st century healthcare [Internet]. Chicago: Healthcare Information and Management Systems Society; [cited at 2011 Jun 1]. Available from: http://marketplace.himss.org/acct618b/Default.aspx?ta bid=44\&action $=$ INVProductDetails \&args $=967 \&$ BookT itle=HIMSS\%20Store $\% 20-\% 20$ Personal\%20Health $\% 20$ Records:\%20The\%20Essential\%20Missing\%20Element\%20in\%2021st\%20Century\%20Healthcare.

6. Amazon. Amazon.com's editorial reviews [Internet]. Amazon: c2011 [cited at 2011 Jun 1]. Available from: http:// www.amazon.com/Personal-Health-RecordsGuide-Clinicians/dp/144433252X/ref=sr_1_1?ie=UTF8 \&qid=1306552450\&sr=8-1.

7. Patients know best. Personal health records: a guide for clinicians [Internet]. [cited at 2011 Jun 1]. Available from: http://wiki.patientsknowbest.com/Our_books/ Personal_Health_Records:_A_guide_for_clinicians. 